\title{
CONTRIBUIÇÃO PARA UMA HISTÓRIA DE OS HERDEIROS - ENTREVISTA COM MONIQUE DE SAINT-MARTIN*
}

\author{
Ana Maria F. Almeida* \\ Graziela Serroni Perosa** \\ MaUricio ERnica ${ }^{* * *}$
}

\begin{abstract}
RESUMO: Nessa entrevista, Monique de Saint-Martin examina o contexto e as operaçóes de pesquisa que cercaram a produção do livro $O s$ Herdeiros - Os estudantes e a cultura. Ela também reflete sobre o lugar desse livro no desenvolvimento da contribuição particular dos pesquisadores e pesquisadoras do Centre de sociologie européenne e do Centre de Sociologie de l'éducation et de la Culture para fazer avançar a nossa compreensão sobre o lugar social da escola nas sociedades contemporâneas
\end{abstract}

Palavras-chave: Les Héritiers; Bourdieu; Passeron; História da sociologia; França.

\section{A CONTRIBUTION TO A HISTORY OF BOURDIEU AND PASSERON'S THE INHERITORS - INTERVIEW WITH MONIQUE DE SAINT-MARTIN}

\begin{abstract}
In this interview, Monique de Saint-Martin examines the context and the research operations that gave origin to the book The Inheritors - French students and their relation to culture. She also reflects on the place of this book in the development of the particular contribution that the researchers gathered at the Centre de sociologie européenne and the Centre de sociologie de l'éducation et de la culture have given to our understanding of schooling in contemporary societies.
\end{abstract}

Keywords: Les Héritiers; Bourdieu; Passeron; History of sociology; France.

\footnotetext{
* Tradução de Ana Maria F. Almeida.

** Universidade Estadual de Campinas, Faculdade de Educação, Campinas, SP., Brasil. E-mail de contato: aalmeida@unicamp.br.

*** Universidade de São Paulo, Escola de Artes, Ciências e Humanidades, São Paulo, SP., Brasil. E-mail de contato: grazielaperosa@yahoo.com.br.

**** Universidade Estadual de Campinas, Faculdade de Educação, Campinas, SP., Brasil. E-mail: ernica@unicamp.br.
} 


\title{
CONTRIBUTION À UNE HISTOIRE DE LES HÉRITIERS - EnTretien AVEc Monique de SAINT-MARTin
}

\begin{abstract}
RÉSUMÉ: Dans cet entretien, Monique de Saint-Martin examine le contexte et les opérations de recherche qui sont à l'origine du livre Les Héritiers: les étudiants et la culture. Elle réfléchit aussi sur la place de ce livre dans le développement de la contribution particulier des chercheurs et chercheuses du Centre de sociologie européenne et du Centre de sociologie de l'éducation et de la culture pour faire avancer notre compréhension de la scolarisation dans les sociétés contemporaines.
\end{abstract}

Mots-clés: Les Héritiers; Bourdieu; Passeron; Histoire de la sociologie; France.

\section{Qual era o contexto institucional quando essa pesquisa foi pensada? De onde veio a ideia? Quem participou? Como o grupo foi constituido e sob qual en- quadramento institucional? $O$ que o trabalho em equipe significava naquele momento?}

O final dos anos 1950 e início dos 1960 constituem, na França, um momento de franco desenvolvimento da sociologia. Esse período marca o início do seu ensino nas universidades. As graduações e os doutorados em sociologia são criados em 1958, primeiro em Paris, um pouco mais tarde no resto do país. As duas primeiras teses são defendidas em 1961. Várias revistas de sociologia são fundadas: Sociologie du travail [Sociologia do Trabalho] em 1959, a Revue française de sociologie [Revista Francesa de Sociologia], em 1960. Iniciativas diversas são numerosas no ensino de sociologia e na pesquisa. Esse é também um período de expansão dos recursos financeiros e humanos dedicados à pesquisa, como nota Philippe Masson (2001).

No Centre de sociologie européenne [Centro de Sociologia Europeia], da École pratique des hautes études (EPHE) [Escola Prática de Altos Estudos], numerosos levantamentos e pesquisas foram desenvolvidos no início dos anos 1960 sobre os estudantes da Faculté de lettres ${ }^{1}$. Pierre Bourdieu, que se tornara professor na universidade de Lille, e Jean-Claude Passeron, que era professor assistente na Sorbonne, ambos agrégés ${ }^{2}$ de filosofia e antigos alunos da École normale supérieure [Escola Normal Superior], foram os principais responsáveis por essas pesquisas. Vários professores universitários colaboraram, principalmente Marcel Maget em Dijon; Guy Vincent em Lyon; Paul de Gaudemar em Toulouse; JeanRené Tréanton em Lille. Eles passaram os questionários em suas classes e também organizaram pesquisas próprias, como foi o caso, por exemplo, de Marcel Maget, sobre o uso 
do tempo pelos estudantes, e de Guy Vincent, sobre as atitudes dos estudantes face aos professores e ao ensino.

Diversos estudantes de sociologia de Lille e de Paris, reunidos nos Grupos de trabalho universitário (GTU) ou trabalhando individualmente, fizeram levantamentos por entrevista ou questionário, mencionados na seção "Advertência" no início do livro. O GTU de Lille se interessou por examinar o interconhecimento entre estudantes; o GTU de Paris pesquisou o grupo de teatro grego da Sorbonne e seu público, e o estudante tal como visto pelos estudantes. Conta Yvette Delsaut (2005, p. 70), que era estudante de sociologia em Lille à época:

\begin{abstract}
Náo sabíamos mais precisamente se se tratava de trabalhos práticos ou de pesquisa pessoal; era, ao mesmo tempo, uma pequena cozinha permanente e um grande projeto [...]. Em Lille, nós não fomos avisados de que existia um projeto de publicar um livro sobre os questionários. Sua publicação nos surpreendeu, mas sobretudo no sentido, eu creio, de que nos parecia curioso que um livro de verdade pudesse sair de nossas sessóes de trabalhos práticos $[\ldots]$.
\end{abstract}

A codificação e a exploração do material foram realizadas por vacataires [assistentes de pesquisa]. Assim, foram muitos os que participaram das diferentes operaçóes, dos estudos preliminares, das investigaçóes e é difícil saber exatamente o número de pessoas implicadas de uma maneira ou de outra na preparação do livro, sobretudo nos diferentes estudos e pesquisas que se entrecruzaram com outras pesquisas e atividades.

Pierre Bourdieu e Jean-Claude Passeron, que dirigiram os levantamentos e pesquisas, escreveram o livro rapidamente. Os Herdeiros, tendo por subtítulo Os estudantes e a cultura, é apresentado, como indicam seus autores na "Advertência" mencionada, como um "ensaio" que se baseia em pesquisa. Alain Darbel, administrador do Institut national de la statistique et des études économiques (Insee) [Instituto Nacional de Estatística e de Estudos Econômicos], escreveu uma "Nota metodológica" sobre as chances escolares e explica como foram calculadas as probabilidades objetivas de acesso ao ensino superior para os estudantes oriundos das diferentes categorias socioprofissionais. E Jacques Bertin, do Laboratoire de cartographie [Laboratório de Cartografia] da EPHE, é objeto de um agradecimento pelas representaçôes gráficas realizadas em seu laboratório.

Eu devo precisar que não participei dos levantamentos e pesquisas que serviram de base a Os Herdeiros. Eu cheguei ao Centre de sociologie européenne (CSE) no outono de 1963, no momento em que Bourdieu e Passeron escreviam o livro, e eu não sei dizer de onde e como surgiu a ideia do livro, sem dúvida progressivamente. Entre as primeiras tarefas que me foram confiadas por Passeron, 
cujas aulas eu seguia na Sorbonne, estava a pesquisa de dados estatísticos fornecidos pelo Bureau universitaire de la statistique [Escritório Universitário de Estatística] sobre a evolução do número de estudantes por universidade, por disciplina, sexo, para o período entre os anos acadêmicos de 1900-01 e 1962-63, assim como sobre a origem social dos estudantes para o ano acadêmico de 1961-1962. No Institut national de la statistique et des études économiques (Insee) foram pesquisados dados sobre a população ativa, sua repartição por categoria socioprofissional no censo de 1962, assim como os dados sobre a evolução da população francesa.

A organização das pesquisas foi, assim, muito diferente do que se tem em mente hoje quando se pensa em um projeto de pesquisa construído e amadurecido, apresentado em resposta a um edital, no qual são previstos, antes de que sejam iniciadas as diferentes operaçóes de pesquisa, as tarefas a cumprir, os participantes, o cronograma. Jean-Claude Passeron nota, aliás, a improvisação e o caráter artesanal dos primeiros levantamentos sobre os quais se apoia Os Herdeiros. (MOULIN; VEYNE, 1996) E Michel Éliard conta como, na sua chegada ao Centre de sociologie européenne, recrutado em 1962 para um posto de técnico no Conseil national de la recherche scientifique (CNRS) [Conselho Nacional da Pesquisa Científica] por Raymond Aron, que não tinha muito trabalho para lhe atribuir, Bourdieu e Passeron propóem ver o que ele poderia fazer sobre os questionários respondidos pelos estudantes de ciências humanas e sociais [cf. nota 1] que estavam então dentro de um armário. (MASSON, 2002, p. 489)

Passeron (2004, p. 23) nos permite imaginar e compreender o espírito no qual foi pensado e escrito o livro. Em um texto escrito após o falecimento de Bourdieu, ele explica que

[...] no total, foi a associação entre um desejo de [realizar] pesquisa científica e uma vontade de esvaziar a ilusão pedagógica dominante na França, as representaçôes da escola, que haviam suscitado em nós a organização de um programa de pesquisas sobre as desigualdades de chances escolares e sobre os fatores sociais e culturais da sua perpetuação [...].

Ele confirma assim a hipótese segundo a qual o livro foi escrito em parte em reação contra as ilusões pedagógicas e políticas, contra as falsas evidências, contra a doxa escolar, que sustentavam os debates da época, e que tratavam como uma evidência estabelecida que "existe alunos 'dotados' e outros 'pouco dotados" e que os "estudantes devem ser selecionados segundo suas 'aptidôes". O livro se distancia também dos trabalhos realizados no Institut national d'études démographiques (Ined) [Instituto Nacional de Estudos Demográficos] por Alain Girard e Henri Bastide, que seguiam um corte de alunos desde o final dos estudos secundários e que insistiam sobre a vontade ou falta de vontade das famílias entre os fatores de sucesso ou fracasso escolar. As desigualdades de chances de acesso ao 
sexto ano $^{3}$ e ao ensino superior segundo os diferentes grupos sociais não são, para Bourdieu e Passeron, apenas o produto de mecanismos externos à escola. Elas estão inscritas no próprio coração do ensino [ou sistema de ensino]. Os Herdeiros, e depois $A$ Reprodução, se opóem à visão encantada da "escola democrática", tal como ela se exprimia, por exemplo, em L'école libératrice [A Escola Liberadora], órgão de imprensa do Syndicat national des instituteurs (SNI) [Sindicato Nacional dos Professores do Ensino Fundamental], que via no ensino de massa (pela implementação de um tronco comum) um meio de lutar contra as desigualdades sociais na escola. A visão encantada da escola era também bastante difundida entre os estudantes. Conta Christian Baudelot em uma entrevista (MOLLE, 2008):

\begin{abstract}
Nós, estudantes, estávamos persuadidos que havia na França pelo menos um lugar de igualdade e promoçáo das chances: a escola laica, gratuita, obrigatória, que garantia a igualdade de chances. Ninguém à época pensava que a escola desempenhava um papel na reprodução das desigualdades sociais [...].
\end{abstract}

Pode-se também lembrar que entre 1954 e 1959, numa França frequentemente indiferente ao conflito argelino, os estudantes foram o primeiro grupo a se mobilizar contra a continuação da guerra e que o movimento sindical estudantil esteve muito ativo no período que se seguiu ao final da guerra. Depois dos acordos de Evian, em 1962, ele se dedica sobretudo às reivindicaçóes internas. A questão da democratização do ensino era central nos debates sindicais. Os autores de $O s$ Herdeiros criticam, aliás, uma das teses amplamente difundidas na época no movimento sindical estudantil, que tendia a considerar a condição estudantil como homogênea, e revelam a variedade de comportamentos dos estudantes em função de sua origem social. Nesse período de crescimento significativo do número de estudantes no ensino superior, se a instituição escolar não era um objeto de pesquisas acadêmicas, tampouco o eram a pesquisa sobre os estudantes e a cultura, a pesquisa sobre a transmissão da herança e aquela sobre as desigualdades diante da escola.

\title{
Como se explica a opção por uma pesquisa por questionário? Havia outras ex- periências desse tipo de pesquisa em andamento na época? Havia especialistas nesse tipo de "técnica" de produçáo de dados? Como os questionários foram construídos?
}

A pesquisa por questionários com a exploração estatística é o método de referência nos anos 1960 na França para os sociólogos tanto no Centre d'études sociologiques [Centro de Estudos Sociológicos] quanto no Centre de sociologie européenne e em outros centros de pesquisa. Não era, no entanto, a única abordagem. 
Os sociólogos não faziam muitas entrevistas e frequentemente não sabiam fazê-las. A pesquisa por entrevistas era de fato pouco desenvolvida e vai ocupar um espaço crescente a partir dos anos 1970. A abordagem etnográfica era pouco frequente. Há apenas alguns exemplos isolados de pesquisas que se apoiavam sobre uma abordagem etnográfica.

No caso de Os Herdeiros, penso ser interessante sublinhar que o livro não se apoia apenas sobre pesquisa por questionários, mas também sobre dados estatísticos em escala nacional, assim como sobre observação de aulas, de provas, de biblioteca e sobre a experiência dos autores. Os extratos de entrevistas são muito breves.

O que me chama a atenção é o caráter um pouco improvisado de certas pesquisas, questionários preparados rapidamente. Com frequência, contudo, eles foram testados, precedidos por pesquisas preliminares, consultas a especialistas para a elaboração de questóes, muitas vezes altamente sofisticadas, por exemplo, sobre os músicos. As questóes e exercícios propostos são bastante diversificados. Quando se tratou de estudar o interconhecimento, propôs-se aos estudantes indicar o seu lugar e o lugar dos estudantes que eles conheciam, sobre um esquema que representava a sala de aula, e de precisar o modo de conhecimento (de nome, de conversas, de atividade em comum). Na pesquisa chamada de "vocabulário", diferentes tipos de exercícios foram apresentados aos estudantes, de definição de termos, de escolha da boa definição de uma palavra entre diferentes definiçóes propostas, ou ainda de localizar numa frase as palavras empregadas de maneira incorreta.

Era durante as aulas que os estudantes deveriam preencher os questionários. Yvette Delsaut (2005, p. 68) conta:

Eu guardo, das sessóes de preenchimento coletivos de diferentes questionários, uma lembrança um pouco sombria. Em Lille, a operação se desenvolveu, em geral, nas aulas mais frequentadas, onde a audiência era mais numerosa, quer dizer, durante as aulas de sociologia dadas pelo próprio Bourdieu, no quadro do curso de "Moral e Sociologia" [...]. Eu me lembro de uma atmosfera bastante tensa. Os filósofos visivelmente consideravam que as perguntas eram grosseiras e redutoras, enquanto outros sofriam e não podiam deixar de se ver submetidos a algum tipo de teste ou exame de conhecimentos. [...] Bourdieu, que queria, sem dúvida, evitar ser tomado como disciplinador e dar ao exercício uma conotação escolar excessivamente estreita [...] permanecia ostensivamente mergulhado nos seus próprios afazeres durante a aplicação, apenas levantando o nariz, de tempos em tempos, com um olhar sorridente que náo parava em nenhum lugar e que poderia dar a entender que se divertia, o que acabava por fazer a atmosfera pesar ainda mais [...]. 


\begin{abstract}
Por fim, e talvez o mais importante, qual foi a importância dessa experiência sobre a formaçáo de jovens pesquisadores e na constituiçáo do trabalbo coletivo desenvolvido no Centre de sociologie européenne? Qual foi o lugar ou a contribuiçáo dos jovens pesquisadores que se integraram à equipe?
\end{abstract}

Os jovens pesquisadores ou estudantes participaram de maneira fragmentada de Os Herdeiros. Apesar disso, eles foram fortemente marcados pelo livro, que se tornou rapidamente emblemático. Christian Baudelot (2008) conta que à época ele seguia com paixão o seminário de Bourdieu e Passeron, na École normale supérieure, consagrado à sociologia dos estudantes e que, no final do último seminário, foi pedir conselhos a Bourdieu. Baudelot já tinha feito na época uma agrégation de letras clássicas, mas como não havia estudado nem sociologia nem filosofia, acreditava-se incapaz de fazer sociologia. Bourdieu o convida, no entanto, a passar pelo Centre de sociologie européenne, o que ele faz rapidamente. Ao chegar lá, foi-lhe atribuído "[...] fazer muitas estatísticas, cálculos de porcentagens, quadros para simplificar agrupamentos etc. [...].” Assim, prossegue Baudelot, "[...] meu primeiro trabalho, minha primeira 'ação' de sociólogo, foi servir de "faz-tudo" [grouillot], foi dar uma 'mãozinha' a Bourdieu e Passeron [...].” (p. 43)

Jovens pesquisadores e estudantes estiveram mais associados a outras publicaçóes que não tiveram a mesma ressonância de Os Herdeiros entre os estudantes. Em 1964 foram publicados na coleção Les cahiers du Centre de sociologie européenne [Cadernos do Centro de Sociologia Europeia], pelas Editions Mouton, Les étudiants et leurs études [Os estudantes e seus estudos], escrito por Bourdieu e Passeron com a colaboração de Michel Éliard (1964) e, em 1965, Rapport Pédagogique et Communication [Relação pedagógica e comunicação], escrito por Bourdieu, Passeron e eu. Nesse segundo caderno, Baudelot publica La rhétorique étudiante à l'examen [A retórica estudantil no exame], que se apoia sobre a análise de 160 dissertaçóes, escritas e corrigidas em Paris nos exames de outubro de 1962 e julho de 1963 dos cursos de Moral e Sociologia e de Sociologia Geral. Guy Vincent escreveu Les attitudes des étudiants face aux enseignants et à l'enseignement [As atitudes dos estudantes face aos professores e ao ensino], que se apoia sobre uma pesquisa realizada em 1963-1964 a partir de um questionário respondido por estudantes de filosofia, sociologia e psicologia, assim como alguns grupos-controle de outras disciplinas. Depois, vários estudantes ou jovens pesquisadores que estiveram envolvidos nessas primeiras pesquisas desenvolveram novas pesquisas sobre os estudantes e as universidades, ou sobre as classes preparatórias e as grandes escolas. É uma longa história e precisaríamos de mais tempo para falar de cada uma dessas pesquisas...

O Centre de sociologie européenne (CSE) foi fundado no início dos anos 1960 e foi lá que as primeiras pesquisas sobre os estudantes e sobre a escola foram realizadas. Muito rapidamente, as tensóes entre Raymond Aron, diretor, e Pierre 
Bourdieu, diretor adjunto, tornaram-se visíveis. Em maio de 1968, a ruptura foi consumada e Bourdieu funda, em 1970, pouco depois dessa separação, o Centre de sociologie de l'éducation et de la culture (CSEC) [Centro de Sociologia da Educação e da Cultura], vinculado à École des hautes études en sciences sociales (EHESS), associado ao CNRS; enquanto Aron dirige o recém-criado Centre européen de sociologie historique [Centro Europeu de Sociologia Histórica], que se extingue quando ele deixa sua direção.

O Centre de sociologie de l'éducation et de la culture (CSEC) foi dirigido primeiro por Bourdieu, entre 1970 e 1984; por Jean-Claude Combessie e eu entre 1985 e 1996; e por Rémi Lenoir em 1997 e 1998, data em que se decidiu reunificar o CSEC com o CSE ou, melhor, de absorver o primeiro no segundo, aliás sem que os antigos diretores do CSEC tenham sido consultados.

De fato, Bourdieu não havia aceitado a ideia do desaparecimento do Centre de sociologie européenne depois da separação de Aron e da criação do CSEC em 1970, e o CSE continuou a existir com o mesmo nome, em paralelo com o CSEC. Quando Bourdieu foi eleito professor do Collège de France em 1982, os membros do CSE juntaram-se a ele, enquanto o CSEC permaneceu no 54 bd Raspail, no prédio da Maison des sciences de l'homme [Casa das Ciências do Homem] e da École des hautes études en sciences sociales [Escola de Altos Estudos em Ciências Sociais]. Depois de 1998, o CSEC desaparece progressivamente da história oficial.

Quais eram os interlocutores desse grupo? As leituras obrigatórias? Como poderiam ser descritas, hoje, as tensóes presentes no momento de construçáo da pesquisa ou de discussáo dos resultados no que diz respeito a usos de Marx e de Weber?

Não há praticamente qualquer referência aos grandes autores nas notas de pé de página de Os Herdeiros. Nas epígrafes, no início de cada um dos três capítulos e na conclusão, são incluídos extratos de textos de Margaret Mead, Emile Durkheim (A evolução pedagógica na França), Hegel e Ernest Renan. No entanto, a inspiração weberiana é claramente reivindicada no livro. Bourdieu e Passeron (1964, p. 89) tentam, principalmente no capítulo 3, intitulado "Aprendizes ou aprendizes de feiticeiro?", construir "o tipo ideal da conduta estudantil em perfeita conformidade com a racionalidade", e explicam que

[...] a racionalização da arte de transmitir a adesão aos valores da cultura, por exemplo no ensino das humanidades ou das disciplinas artísticas, não é mais inconcebível, afinal, do que a racionalização da vida religiosa, no sentido atribuído a ela por Max Weber [...]. (p. 105) 
Além disso, Bourdieu e Passeron ensinavam Weber nos seus cursos. Quanto a Marx, ele não é nem citado, nem evocado diretamente no livro, mas pode-se pensar que ele é uma fonte de inspiração, se levamos em conta a abordagem geral do livro em termos de relaçóes de classe. Quanto ao peso da "herança" cultural, a ideia veio, segundo Passeron (2004), de Saint Simon. Escreve ele:

\begin{abstract}
No que me diz respeito, foi a leitura das diatribes de Saint Simon contra a mecânica reprodutiva dos privilégios da fortuna transmitidos pela herança dos patrimônios econômicos, descrito como o principal obstáculo à liberaçáo das energias produtivas em uma sociedade industrial, que me serviu de sugestáo para a extensão analógica da noção de herança ao papel desempenhado pela transmissão de um patrimônio (ou de um capital) cultural.
\end{abstract}

No final dos anos 1950, os trabalhos anglo-saxôes sobre a instituição escolar não eram tão numerosos e não fizeram parte dos autores de referência de Os Herdeiros. No entanto, Bourdieu e Passeron se apoiaram, explicitamente, nos trabalhos do sociólogo inglês Basil Bernstein sobre as diferenças de uso da linguagem entre as diferentes classes sociais. Pode-se notar, também, as referências explícitas aos trabalhos de uma socióloga húngara, Zsuzsa Ferge, sobre as chances escolares na escala do grupo familiar e de um sociólogo polonês, Jan Sczepanski, sobre a origem social dos estudantes na Polônia. Bourdieu e Passeron estavam de fato interessados na ideia de comparação de diferentes sistemas de ensino e se interessavam sobre a questão da democratização do ensino nos países, então, socialistas.

Na sua avaliaçáo, qual foi o lugar de Os Herdeiros no desenvolvimento de um pensamento sobre a escola no seio do CSE e do CSEC? Parece-nos importante situar o livro em relaçáo às obras que vieram depois, sobretudo sabendo que o modelo teórico apresentado em "A Reproduçáo" náo estava desenvolvido ali (ainda?).

Os Herdeiros constitui, sem dúvida, o ponto de partida de numerosas pesquisas e análises sobre os estudantes e sobre o sistema de ensino realizadas no CSE e depois no CSEC. Ali se combina uma pesquisa empírica e uma análise bastante abstrata da instituição escolar a partir de sua função de legitimação da ordem social, que será mais amplamente desenvolvida em seguida. Os trabalhos seguintes tentarão, da mesma forma, aliar a pesquisa empírica, os levantamentos e o questionamento teórico, e devem muito às hipóteses e às análises de Os Herdeiros, sejam as pesquisas de Claude Grignon sobre o ensino técnico, sejam as de Yvette Delsaut sobre a ideologia dos estudantes das Facultés des lettres [cf. nota 1] ou aquelas que desenvolvi com Robert Castel, Madeleine Lemaire e outros colegas sobre o ensino científico superior. Assim que dou início às pesquisas sobre os 
estudantes das facultés des sciences ${ }^{4}$, a questão que me coloco é de saber se o ensino nas ciências [exatas e biológicas] é tão neutro socialmente quanto se pensava frequentemente, ou se náo se observa ali as diferenças nas práticas e julgamentos dos estudantes associadas, pelo menos em parte, à origem social.

É sem dúvida em $A$ Reprodução que a escola se torna objeto central da análise sociológica. Esse livro constitui uma tentativa de apresentar os fundamentos de uma teoria geral das açóes de violência simbólica e das condiçóes sociais de dissimulação dessa violência. Muitos dos temas e das análises que se encontram ali haviam sido abordados anteriormente como, por exemplo, os fatores sociais da comunicação pedagógica, o significado dos exames, a questão dos objetivos da educação escolar. Mas $A$ Reprodução não é a repetição das análises anteriores. O subtítulo Elementos por uma teoria do sistema de ensino indica claramente que a problemática é teórica. O objeto do livro é construir ou contribuir para a construção de um "modelo" que permita compreender o funcionamento do sistema escolar e sua função social real, apoiando-se em pesquisas empíricas

Nos anos 1960 e início dos anos 1970, depois dos primeiros trabalhos, as pesquisas desenvolvidas pelos pesquisadores reunidos em torno de Pierre Bourdieu e de Jean-Claude Passeron se expandiram para abarcar os estudantes de diferentes faculdades: lettres [cf. nota 1], direito, medicina, sciences [cf. nota 4], assim como aos estudantes e professores das classes preparatórias para as grandes escolas, e em seguida, as grandes escolas etc. Essas pesquisas indagaram sobre as funçóes desempenhadas pelo sistema de ensino: reprodução, transmissão de conhecimentos, seleção, eliminação, classificação etc., e sobre as relaçôes de concorrência e de dominação existentes entre as diferentes instituiçóes de ensino, assim como sobre as relaçôes entre o sistema de ensino e o mercado de trabalho.

A confrontação permanente das observaçóes, materiais de pesquisa e interrogaçóes teóricas dava origem a novas questóes e novas pesquisas. Assim, a análise de uma pesquisa junto aos premiados do concurso geral e, depois, das avaliaçóes de trabalhos por uma professora de classe preparatória nos conduziu a um estudo dos critérios implícitos e explícitos do julgamento professoral, que levou, por sua vez, a um exame das notas póstumas dos estudantes da École normale supérieure e, a partir disso, a um estudo da moral e das virtudes universitárias. (BOURDIEU; SAINT-MARTIN, 1970; 1975)

\section{Em "Os Herdeiros", os autores criticam a "pedagogia carismática" e propóem uma "pedagogia racional". Qual foi o lugar dado a essa ideia posteriormente? Ela foi de algum modo modificada?}

O projeto de uma pedagogia racional ou, ainda, de uma pedagogia do "contre-handicap" [contra a deficiência] de inspiração sociológica é associada, com 
justiça, a Os Herdeiros e influenciou as reflexóes pedagógicas posteriores. A criação das Zones d'éducation prioritaires (ZEP) [Zonas de educação prioritária] nos anos 1980 lhe deve sem dúvida alguma coisa. Havia uma grande preocupação de que fosse possível, a partir das análises sociológicas realizadas, obter orientaçóes para a pedagogia (PASSERON, 2004, p. 29)

O contexto intelectual no qual foi escrita $A$ Reprodução, alguns anos mais tarde, logo após os acontecimentos da primavera de 1968, era bem diferente. A radicalidade universalista das teses desenvolvidas nessa obra deve ser aproximada de outras teses que foram desenvolvidas no mesmo momento, algumas inspiradas pela política escolar implementada na China e outras pelas obras de Michel Foucault. Essa mudança no contexto político explica sem dúvida, segundo Jean-Michel Chapoulie (2005, p. 04), o desaparecimento da defesa de uma "pedagogia racional” presente em Os Herdeiros, que aparece em A Reprodução sob o rótulo, mais modesto, de "pedagogia explícita".

\section{Nesse quadro, que tipo de problema a noçáo de reconversão pode resolver?}

As primeiras obras e as primeiras pesquisas haviam sublinhado a força reprodutora da escola, sua função conservadora, o peso da herança cultural, e não há dúvida de que era necessário dissipar as ilusóes. No entanto, tornava-se claro ou mesmo evidente, sobretudo com os acontecimentos de maio 1968, que mudanças estavam acontecendo no sistema de ensino e que grupos importantes de estudantes e de professores eram favoráveis às transformaçôes, o que não significa dizer que as forças conservadoras haviam desaparecido de uma hora para outra. A crise de maio de 1968 nos levou a desenvolver um vasto conjunto de pesquisas sobre as transformaçóes do sistema de ensino, sobre as reaçôes à crise, sobre as transformaçóes da sociedade, demandadas ou reivindicadas por diferentes grupos: professores, pais de alunos, estudantes, alunos (cf. o estudo desenvolvido por Pierre Bourdieu, Luc Boltanski, Yvette Delsaut, Pascale Maldidier e por mim). Falava-se então de fatores de mudança e das forças da inércia no sistema de ensino. Buscava-se compreender as mudanças associadas às transformaçôes do sistema de classes sociais e aos deslocamentos observados. Entre as mudanças observadas, estava a diminuição relativa dos agricultores, dos artesãos e dos pequenos comerciantes na estrutura das classes sociais, que renunciavam, às vezes, a transmitir seu patrimônio econômico a seus filhos.

Nos anos 1960 e 1970, as reconversóes observadas e analisadas na França aconteciam frequentemente por meio da escola. Os industriais, os grandes comerciantes, os artesãos, os comerciantes e os agricultores constituíam grupos que se engajavam - frequentemente em duas gerações - em reconversóes de capital ou de recursos econômicos, de ativos transmissíveis pela herança em capital ou em recursos escolares, em investimentos educacionais. ${ }^{5}$ 
Operar uma reconversão é, para os membros de uma classe ou de uma fração de classe, operar "[...] uma mudança de estratégias e de instrumentos de reprodução destinados a reproduzir ou elevar sua posição pelo abandono de sua condição [...]", escrevíamos no artigo publicado em 1973 (BOURDIEU; BOLTANSKI; SAINT-MARTIN, 1973, p. 101). As estratégias de reconversão estavam, assim, inscritas no conjunto das estratégias de reprodução, sem que se falasse propriamente em estratégias de reprodução, já que a mudança era o seu fundamento. "Mudar para se manter" tornava-se necessário.

\section{O artigo "Une bonne éducation" [Uma boa educaçáo] foi publicado em 1990. Em que momento a ideia de estudar a escola privada tornou-se interessante? Epor quê?}

As primeiras pesquisas tratavam principalmente das universidades públicas e não estavam muito preocupadas com as universidades católicas, tão raras na França, que acolhiam apenas uma fração ínfima dos estudantes. Na pesquisa sobre as grandes escolas, iniciada em 1966, escolas públicas e privadas, por exemplo, a École central [Escola Central] ou a École des hautes études commerciales (HEC) [Escola de Altos Estudos Comerciais], foram levadas em conta sem, no entanto, que se discutisse substantivamente o que poderia distinguir as escolas públicas das escolas privadas.

É apenas quando as pesquisas sobre o ensino secundário passaram a se desenvolver no CSEC que o interesse pelo ensino privado aparece nas análises e nos programas. Assim, o relatório de atividades do CSEC para o CNRS, relativo aos anos de 1976 e 1977, na parte dedicada às "estratégias educacionais", intitula uma subparte como "Ensino público e ensino privado: as estratégias de transmissão cultural”, que se apoia em questionários e entrevistas, realizados por Jean-Claude Chamboredon e François Bonvin numa região da periferia parisiense (Yerres, no departamento de Essonne). Os dois pesquisadores recensearam o sistema quase completo da oferta escolar, isto é, eles se interessaram pelo conjunto de escolas, públicas e privadas, implantados nessa região. François Bonvin, que havia realizado um estudo monográfico muito aprofundado de uma escola privada dessa região, publicou pouco depois um artigo que marcou época. (BONVIN, 1979)

Em seguida, Jean-Pierre Faguer (1991) estudou o colégio Franklin em Paris, no 16ème arrondissement [16o. distrito], colégio de jesuítas. Ele se interessou pela pedagogia ali implementada, pelas trajetórias de uma geração de alunos, estratégias matrimoniais e divisão do trabalho entre casais. Nesse colégio, como aliás na escola des Oiseaux para moças que eu estudei pouco depois, e que deu lugar ao artigo "Une bonne éducation" [Uma Boa Educação], os alunos eram mantidos à distância de toda possibilidade de contato com alunos oriundos de outras classes sociais, não apenas durante as horas de aula, mas também durante a maior parte 
das atividades de lazer dirigidas pela escola. (SAINT-MARTIN, 1990) Eu me interessei por essa escola privada para moças, em grande parte, pela pesquisa que desenvolvia sobre os descendentes da antiga nobreza, especialmente sobre suas estratégias educativas e de reconversão. A escola des Oiseaux acolhia alunos oriundos tanto da burguesia quanto da aristocracia. A escolha das escolas secundárias públicas ou privadas apareceu, aliás, como algo decisivo para os proprietários das maiores empresas francesas quando fizemos a pesquisa sobre o patronato. Não eram as mesmas frações da burguesia que frequentavam umas e outras. (BOURDIEU; SAINT-MARTIN, 1978)

\section{Notas}

1. N.T.: A pesquisa que deu origem a Les héritiers foi realizada entre os estudantes de Faculté des lettres em diferentes universidades. A Faculté des lettres incluía, à época, os cursos de literatura, língua antiga e moderna, filosofia, história, geografia, sociologia, música, psicologia, entre outros, assemelhando-se ao que conhecemos no Brasil como "ciências humanas e sociais".

2. N.T.: Tendo sido aprovados no competitivo concurso para professor do ensino secundário e superior público francês, a agrégation.

3. N.T.: Na França, o sexto ano é a primeira série após os estudos primários, marcando o início do primeiro ciclo dos estudos secundários (chamado de collège).

4. N.T.: As facultés des sciences reuniam os estudantes matriculados em ciências matemáticas, ciências físicas e ciências naturais .

5. As pesquisas se interessavam prioritariamente pelos processos de reconversão do capital econômico em capital cultural certificado ou em capital escolar, na sequência principalmente das transformaçốes do campo das empresas e do mundo agrícola.

\section{Referências}

AGACINSKI, D. Comment la guerre d'Algérie a politisé le milieu étudiant. Cahiers $d u$ GRM [On-line], Toulouse, 3, 2012, Disponível em: <http://grm.revues.org/258>. Acesso em: 29/06/2014.

BONVIN, F. Une seconde famille. Un collège d'enseignement privé. Actes de la recherche en sciences sociales, Paris, v. 30, 1979, p. 47-64.

BOURDIEU, P.; PASSERON, J.-C. Les héritiers. Les étudiants et la culture. Paris: Ed. de Minuit, 1964.

BOURDIEU, P.; SAINT-MARTIN, M. de. L'excellence scolaire et les valeurs du système d'enseignement français. Annales, XXV, 1, 1970, p. 147-175.

. de. Les catégories de l'entendement professoral. Actes de la recherche en sciences sociales, 3, 1975, p. 68-93.

de. Le patronat. Actes de la recherche en sciences sociales, 20-21, 1978, p. 3-82. 
BOURDIEU, P.; BOLTANSKI, L.; SAINT-MARTIN, M. de. Les stratégies de reconversion. Les classes sociales et le système d'enseignement. Information sur les sciences sociales, v. 12 , n. 5, 1973, p. 61-113.

CHAPOULIE, J.-M. Sur le contexte des Héritiers et de La Reproduction. In: CHAPOULIE, J.-M. et al. (Dirs.). Sociologues et sociologies. La France des années 1960, Paris, L'Harmattan, 2005.

. Un regard rétrospectif sur un demi-siècle d'enquêtes empiriques dans la sociologie française. Education et sociétés, 2012, v. 2, n. 30, p. 33-48.

DELSAUT, Y. Sur Les Héritiers. In: CHAPOULIE, J.-M. et al. (Dirs.). Sociologues et sociologies. La France des années 1960, Paris, L'Harmattan, 2005, p. 65-78, 2005. [Traduzido para o português em Depoimento sobre Les Héritiers, Tempo Soc., São Paulo, v.17, n.1, p. 211-228, 2005].

FAGUER, J.-P. Les effets d'une 'éducation totale'. Un collège jésuite, 1960. Actes de la recherche en sciences sociales, v. 86-87, p. 25-43, 1991.

MASSON, P. La fabrication des Héritiers. Revue française de sociologie, v. 42, n. 3, p. 477-507, 2001.

MAUGER, G. Sur 'l'idéologie du don'. Note de recherche. Savoir Agir, v. 17, p. 33-43, sept. 2011

MOLLE, G. Une rencontre avec Christian Baudelot et Stéphane Beaud. Idées économiques et sociales, v. 4, n. 154, p. 42-49, 2008.

MOULIN, R.; VEYNE, P. Entretien avec Jean-Claude Passeron. Un itinéraire de sociologue. Revue européenne des sciences sociales, XXXIV, n. 103, p. 275-354, 1996.

PASSERON, J.-C. Le sociologue en politique et vice versa: enquêtes sociologiques et réformes pédagogiques dans les années 1960. In: BOUVERESSE, J.; ROCHE, D. La liberté par la connaissance, Pierre Bourdieu 1930-2002. Paris: Odile Jacob, 2004, p. 14-104.

SAINT-MARTIN, M. de. Une 'bonne' éducation. Ethnologie française. T. 20, v. 1, pp. 62-70, 1990.

Recebido em 25 de agosto de 2014.

Aprovado em 19 de janeiro de 2015. 